\title{
Environmental Media: Accessing Virtual Representations of Real-time Sensor Data and Site-specific Annotations Embedded in Physical Environments
}

\author{
Scott S. Fisher \\ Keio University at Shonan Fujisawa, Japan

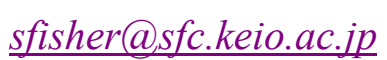

\begin{abstract}
The goal of this research is to explore potential applications of location-based information and communication services over wireless networks with the intention of enabling a mobile user to browse a spatially correspondent multimedia information database about a specific location as it changes over time, to post location-specific information, or to access interpretive annotations posted by domain experts. This paper describes the design and prototyping of a prototye "environmental media" system for linking virtual environments to the physical world, current research to develop comprehensive $3 D$ representations of specific locations derived from both mobile and static real-time wireless sensing devices, and methods for capture, organization, and visualization of real-time, site-specific environmental information for users who are both remote and local to the site. The overall aim is to develop Environmental Media capabilities to facilitate and enhance visualization of natural processes, cycles, and systems by displaying pertinent contextual information that is digitally connected to actual locations in the physical world
\end{abstract}




\section{Introduction}

In 1994, Paul Milgram and colleagues from University of Toronto and colleagues at the Advanced Telecommunications Research Institute International (ATR) in Japan were some of the first researchers to articulate taxonomy for the emerging continuum of virtuality evolving out of work in the areas of interactive computer graphics and human-machine interaction [1].

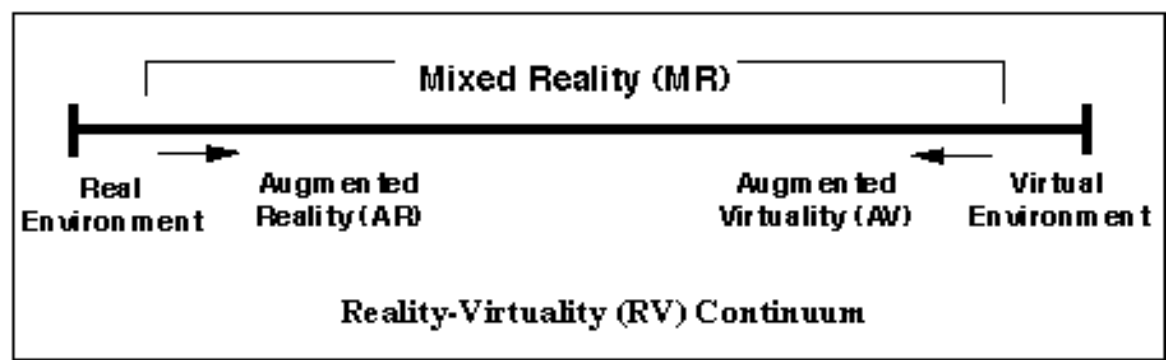

Figure 1. Reality-Virtuality Continuum (Milgram, 1994)

At one end of this continuum appear what have come to be called virtual environments (Figure 1). This mediated experience is typically represented by an image of a user wearing a head-mounted display that enables immersion in a virtual space completely separate from the physical environment. It's taken about 20 years for these VR technologies to emerge from the research laboratory and to be used in a number of different application areas. In spite of unfortunate and misleading representations of VR in movies like "Lawn Mower Man", "Virtuosity", and "Disclosure", currently we can find strong applications of VR technologies in many different disciplines. For example, VR is being used for performing eye surgery in which the surgeon is actually wearing a head-up display or head-mounted display to control a remote device doing retinal repair.

The other end of this virtuality continuum represents the physical environment, and along this axis a new capability is starting to develop that is called augmented reality. In this experience, virtual imagery is combined with or superimposed onto a user's physical environment. The idea of augmenting a real space with virtual objects has been developed primarily by the military and implemented in head-up displays used in fighter aircraft to 
superimpose graphics on to the real world in, for example, zero- or low-visibility conditions. This technology has been commercially adapted for similar head-up displays in automobiles that have just started to become commercially available in the last few years. More recently, it is becoming possible to provide the technology capability of augmented reality for a mobile user to access datasets of information from live sensors and other kinds of location-specific data as they're interacting with their everyday surroundings. This paper will describe developments in a new area of research called Environmental Media that attempts to integrate several new digital media to link virtual environments to the physical world.

\section{Information in places}

Much of the work that has been done in the medium of virtual reality has involved information about places; a simple example is the virtual simulation of physical environments that are used for training, education, and entertainment and in which the user is separate from the physical world that they interact with every day. An early multimedia example is the Aspen Moviemap Project that was developed by the Architecture Machine Group at MIT in the late '70s [2].

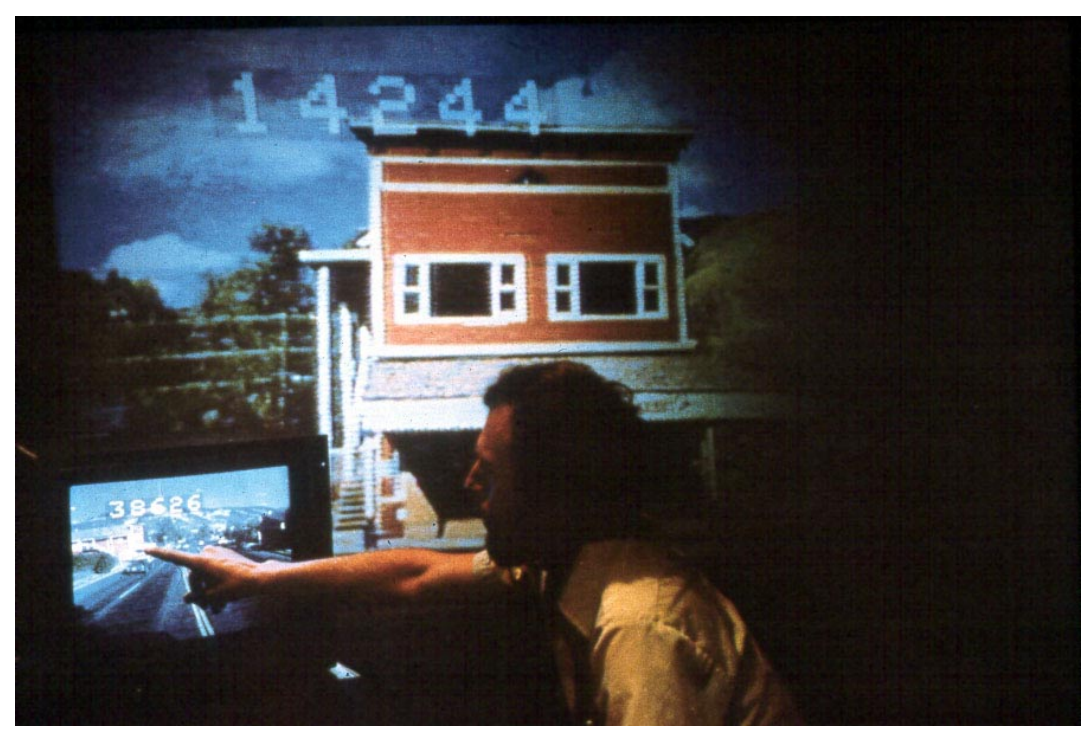

Figure 2. Aspen Movie Map Project, MIT Architecture Machine Group 
In contrast, an important new technological development is the capability for embedding information into the spaces around us. In this scenario, we don't have to sit in a lab or a game center to access virtual space. Instead, we can start to look at the data as we move about in an actual physical space. Of course this isn't exactly a new idea - there are many examples of analog data embedded in the environment around us that we interact with every day - such as signs, advertising, architecture, and so on.

What's also becoming common is that digital data is now becoming embedded in our everyday surroundings - specifically in the form of "location-based services". This has been called ubiquitous computing or pervasive computing. For example, hand-held computers and PDA devices can now be used for accessing location-based services to answer questions such as "Where is a restaurant near to where I am now?", "Where are points of historical interest?", "Where can I find driving directions?" The recently emerging concept of a "smart house" is another venue for embedding digital data, specifically about how to use the house services, data about the person who lives in the house, and so on. And also becoming more common is the idea of location-embedded data for driving information. We're all familiar with signs along the highway that alert you to turn your radio to a particular channel to find out about driving conditions in the vicinity - essentially using the medium of radio to decode information about that specific area. The new GPS-based car navigation systems take this idea several steps further and can provide very detailed maps of location, destination, and pointers to many kinds of services or travel information available to you as you're driving.

As a next step, we can start to envision how these "layers" of data in local areas around us are extending to become what Bell Labs has called a "communication skin", in which many streams of real-time information and millions of electronic measuring devices, are starting to aggregate as huge amounts of data that will be available to us as we move through our environments. For example, in the state of New York in the U.S., there's a national forest that's been instrumented with many real-time data sensor stations from which data about local environmental conditions is sent back in real-time to a server and then posted on the Internet [3]. Similarly, in the state of Oklahoma, there are 114 sensor towers sending data in real time back to one central server to generate visualizations of environmental data for the whole state of 
Oklahoma [4]. Similarly, the Jet Propulsion Lab (JPL) is working under NASA contract to develop networked sensor capabilities where very small sensors, about the size of a golf ball, can be deployed quickly and easily throughout a new environment, such as an alien planet surface, and report back real-time data about environmental conditions there [5].

\section{Key technologies for Environmental Media}

With countless developments like these, the amount of real-time data available to us about specific locations is increasing rapidly. A next major challenge then, is how to visualize this data in a way that is meaningful and useful for human users as they navigate through and interact with these environments. This is one of the objectives of the Wearable Environmental Media project (WEM) at Keio University's Shonan Fujisawa Campus [6]. Some of the technologies used for this project include these three: augmented reality or mixed reality; wearable computing; and mobile multimedia. Augmented reality is becoming a familiar new technology that can be experienced in game centers and entertainment venues like Disneyland's haunted mansion exhibit, where in fact the virtual characters presented there are superimposed onto the real world in front of the visitor by means of a half-silvered mirror or lighting tricks with a large sheet of glass - an old theater illusion called "Pepper's Ghost”. Researcher, Ken Knowlton used a similar system for making virtual keyboards at Bell Labs in the '70s [7]. The University of Tokyo and many other research groups have looked at medical applications for augmenting data onto a patient in real time for the doctor to examine. And, as mentioned earlier, many military and commercial aircraft now have augmented reality displays.

In the U.S., Columbia University and Carnegie Melon University have done much of the pioneering research work in the area of augmented reality, as has Sony CSL research laboratory in Japan. A more recent contribution has been from the Mixed Reality Systems Lab (MRSL) in Yokohama, Japan where over the last four or five years researchers have developed some of the most advanced augmented reality research and applications to date with sponsorship from the Japanese Government and Canon Corporation. MRSL has developed several augmented reality game systems such as "RV Border Guards" and "Aqua Gauntlet" [8]. Players can see 
high resolution virtual computer-generated characters superimposed into their physical game space and the virtual objects actually can also appear behind the real objects on a table - this occlusion capability is a very difficult calibration problem in any augmented reality system and Mixed Reality Lab has developed unique software to enable this illusion. The Advanced Telecommunications Research Institute International (ATR) in the Kansai area of Japan is also doing some interesting work in this area in collaboration with the University of Washington. One compelling example is their Shared Space Project, which is now being used in a "virtual book" where children can open an actual book, see 3-dimensional characters appear, and then enter into the virtual space depicted in the book [9]. A related technology that is being used for the WEM Project is wearable computing, a broader field that involves the integration of displays, PCs, and, wireless network connections in equipment packages that are small enough for a user to wear or carry comfortably at all times.

And finally, the WEM Project incorporates mobile multimedia capabilities - specifically the increasing ability for mobile phones to deliver very high bandwidth multimedia data. NTT DoCoMo is one of the main developers and providers of this service in Japan and a primary objective of the WEM Project is to look at what fourth and fifth generation mobile multimedia service capabilities might be available on such a mobile phone network. There are also many new technologies in development to enable mobile multimedia such as "Bluetooth", a popular new technology that can enable, for example, a personal area network in which a user is surrounded by a 30 -foot sphere of connectivity and can communicate wirelessly with other local devices and services using their mobile phone, digital camera, or wearable computer. An early commercial product example is Ericsson's new Bluetooth-enabled phone where the headset transmits wirelessly back and forth to the handset that could remain in a user's briefcase or pocket.

\section{Research approach and assumptions}

This project is based on several main assumptions about the projected near-term development and evolution of the worldwide technology and communications infrastructure: 


\subsection{Technology capabilities will improve rapidly}

Within the next decade, the basic hardware and system components necessary to implement this system will improve in performance, resolution, and cost by orders of magnitude. Of particular importance are:

- Head-mounted display technologies will have much higher resolution and become lighter weight in a low profile design like regular eyeglasses.

- Mobile phone technology will communicate wirelessly with other devices at high data rates to enable multimedia communication.

- Miniature radio ID tags will be included in all manufactured objects that point to an internet based database of information about where, when, how, and by whom the object was made along with product information and instruction manuals.

- High bandwidth wireless networks will become widely available.

\subsection{A worldwide "communication skin" will grow rapidly}

Information about almost everything in our everyday surroundings is becoming encoded in digital form and is rapidly becoming associated with or embedded in our environment. The combination of this data with proliferating real-time environmental sensing devices, and the expanding communications network, will result in a worldwide layer of site-specific information that Bell Labs has called a "Communication Skin".

\subsection{Increasing interest in "non-formal", informal, and lifelong learning}

These advances in communication and visualization technologies will result in the ability for a mobile user to essentially "browse" an environment, obtain site-specific information, or access representations of real-time data about a location - this will in turn provide a new capability for unique out-of-classroom educational opportunities available to anyone, at anytime with the added benefit of being embedded in the rich context of specific places. 


\section{Interface design issues and examples}

The technologies mentioned above are now all generally available and even becoming fairly low-cost. But an important research and design question remains: How can we integrate these technologies in a way that's useful for an average user? And as mentioned earlier, a main goal of this particular project is: How do we link virtual environments to the physical world? But more importantly, a further question that needs to be explored is: What are the interfaces for these new capabilities and these new services that might be provided by a fourth generation mobile phone?

At the University of Oulu in Finland, recent research has focused on developing interaction concepts for the use of multi-media and a mobile phone in potential future augmented reality scenarios. Their "Cy-Phone" mockup has the typical capabilities of any mobile phone, but proposes some interesting additional capabilities [10]. For example, if a user needs to find a nearby printer to print out a fax he's just received on his mobile phone, he can use the phone as a kind of binocular viewing system, where the computer graphics overlaid on the real world point out available services for him in that immediate space. In other scenarios he uses the phone to pay for a cab ride, and the phone also negotiates a hotel reservation for him, getting data from a sign that the cab passes by as they're driving to his next meeting.

Similarly, in the WEM Project the aim is to actually provide these capabilities through the combination of high-bandwidth wireless network and high-resolution tracking technologies to develop a Wearable Environmental Media platform (WEM) that can display a wide range of location-specific and context-sensitive information to a mobile user in an exterior environment. A first step has been to develop a prototype hardware platform in order to try out several different applications and implementations. Figure 3 illustrates the WEM Project prototype mobile system developed at Keio SFC. The user is wearing a wireless backpack containing a number of different technologies for capturing video imagery, transmitting video and data, and determining user location. The large disk is a GPS antenna to locate the user's location, to about 2-centimeter accuracy. 

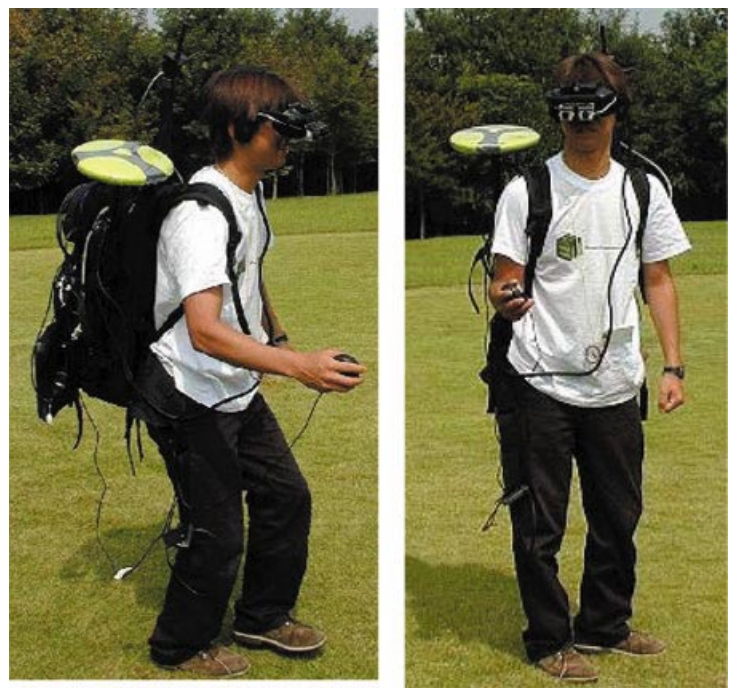

Figure 3. WEM system user accessing multimedia data objects in the test site.

One of the target applications that have been developed to look at this question of interface and interaction requirements is called the "Virtual Field Guide" [11]. In this application scenario, the user can access photographic and computer-generated information about plant and insect forms that are specific to the test site as well as video clips about history of the site. While traversing the site with the system, the user can see many $3 \mathrm{~d}$ icons superimposed onto the location that indicate where information has been embedded. To select the icon, the user aligns a virtual $3 \mathrm{~d}$ cursor over it with the hand-held $3 \mathrm{~d}$ input device. Upon selection, an image based, $3 \mathrm{~d}$ computer generated menu shows additional information that can be chosen. For example, an icon embedded in a tree contains information about a kind of insect that typically lives there. Typical data available includes a 3d CG model, a diagram of its life cycle, a video clip of egg laying, an audio clip of its sound, etc. Other icons in the site point to similar information about other insects or plant forms. In future versions, a user will also be able to investigate changes in vegetation though different seasons by comparing the actual view with previously captured images from the same viewpoint, or view real time visualizations of environmental variables from sensors on the site. So far, a small area on SFC campus has been developed as a test bed site where multi-media data objects have been embedded in the form of computer generated menus, 3Dcomputer graphic models, video, audio, and so on. A future project objective is to use this real-time, site-specific augmentation capability in an exterior unstructured environment 
to help users better understand and visualize the processes and cycles that occur in natural settings around them [12].

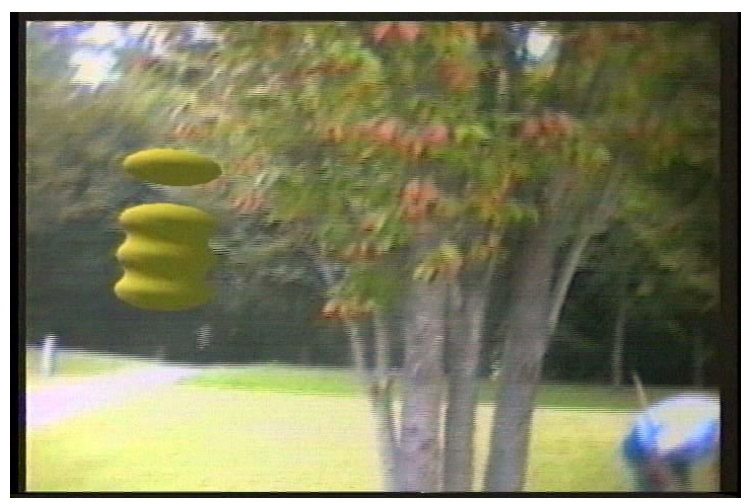

Figure 4. 3D icon superimposed onto test site

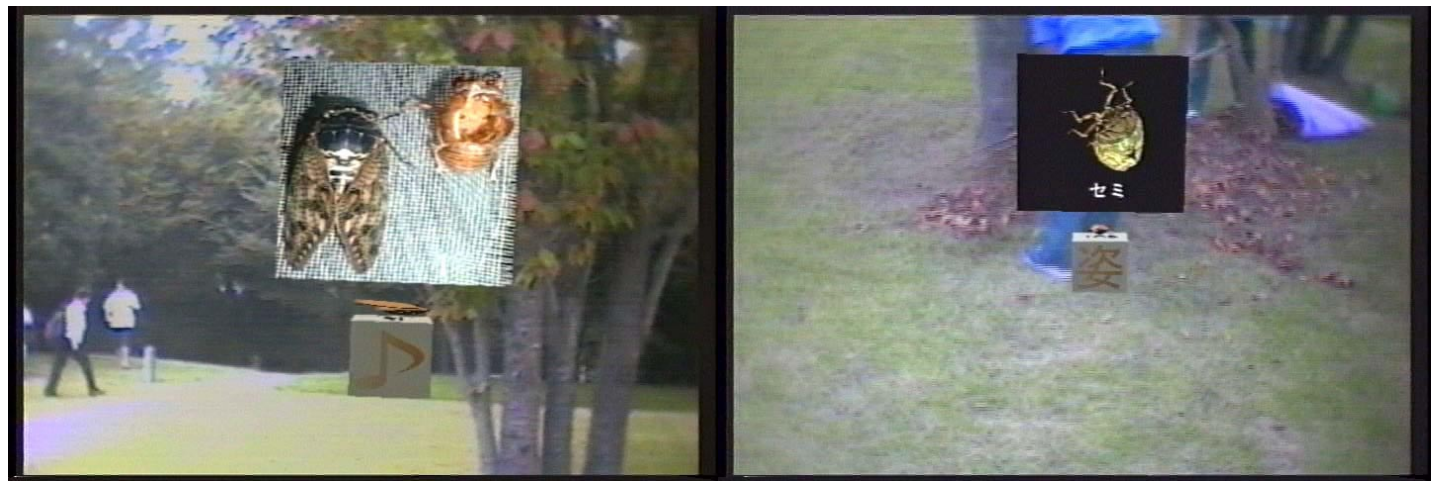

Figure 5. 3D Submenus with life cycle and identification data

\section{WEM system configuration and development}

The primary emphasis of this project is on the development of methods for organization and presentation of site-specific information to both remote and on-site users, and on development of user-interface configurations and software "authoring" tools for linking information to specific locations. As a test bed to evaluate these concepts and configurations, an initial technology platform has been developed that consists of a very lightweight stereoscopic camera and display system with wireless audio/video transmission that is mounted on a remote users head and body. Additional subsystems are added for: presenting audio information; tracking the 
user's location and head orientation; controlling a virtual $3 \mathrm{~d}$ cursor; accessing and caching data about the environment (both archived and local sensor data); and configuring or generating data to be displayed.

Figure 6 shows a picture of the WEM research lab where data from remote users and sensor stations is transmitted back to a dual-channel Intergraph Zx10 workstation using wireless analog video, processed and then sent back to the user in a field nearby our laboratory. Figure 7 illustrates some of the hardware components used in the WEM system. Currently we are using analog video transmission since wireless LAN technology used for digital data transmission are not capable of sending full-frame video at acceptable resolutions and frame rate. The head-mounted display has two CCD cameras mounted on it, from which the stereoscopic video signal is fed back into the display after the augmented graphics are added to it. Also included are GPS rover and base stations from JAVAD, a 3D input device, and an RFID tag reader - all interfaced to a notebook PC in the user's backpack.
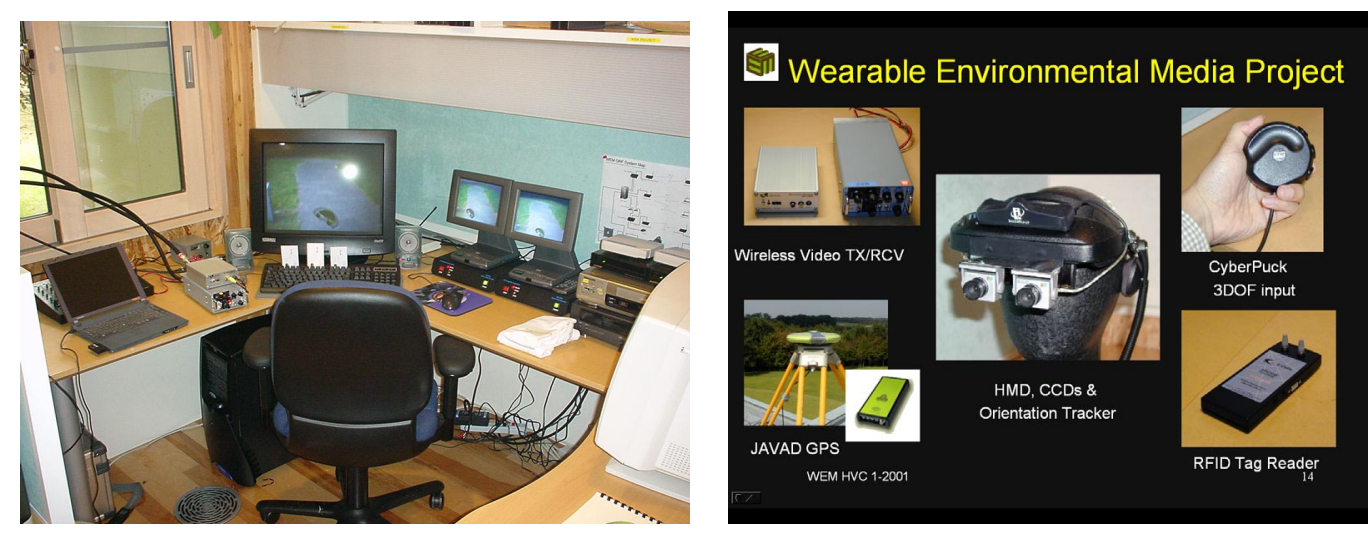

Figure 6.(left) WEM lab with equipment for transmitting data to remote user Figure 7. (right) Current WEM hardware System and components

An important future near term objective for the project is to replace the heavy backpack and the uncomfortable head-mounted display in the prototype system. New display capabilities are now becoming commercially available in which the optics are actually embedded in a normal eyeglass frame (figure 8), or in which unique new approaches in retinal scanning display 
technology are used to scan images directly on to the eye with a low powered laser. In addition the project plans to replace the heavy backpack of equipment with a next generation mobile phone that will be able to handle all of the information transmission and reception from the local servers over high bandwidth wireless connections.

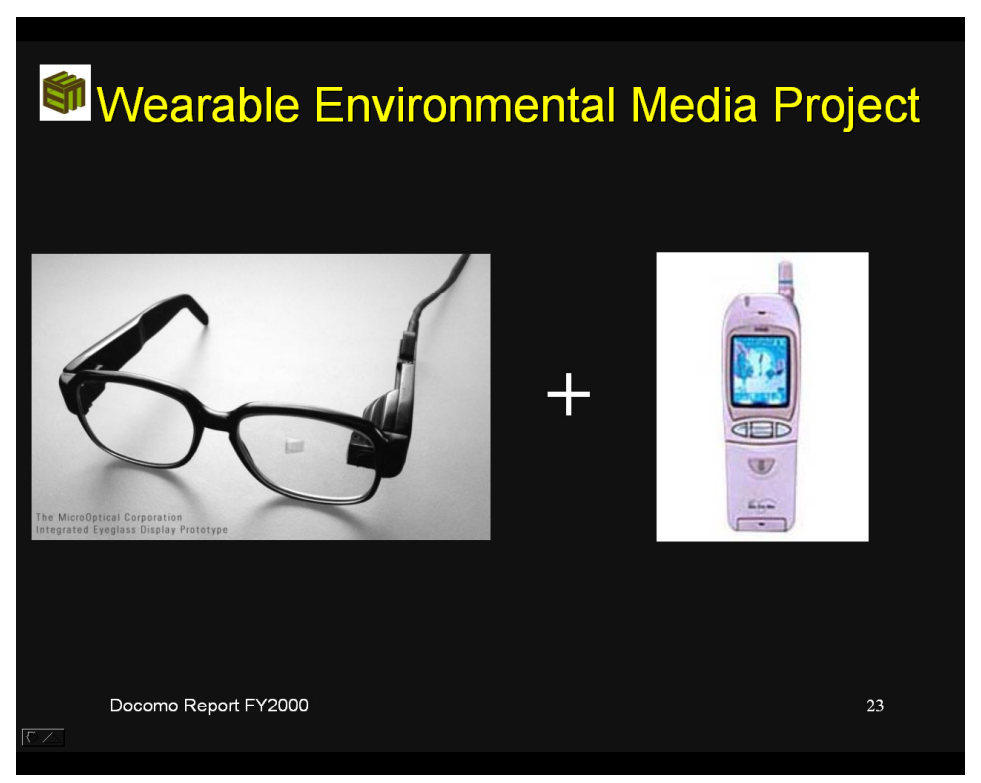

Figure 8. Target configuration for future WEM system

\section{Environmental Data Gathering}

A more recent objective of this research is to develop comprehensive 3D representations of specific locations derived from real-time wireless sensing devices. The specific objectives are to develop methods for capture, organization, and visualization of real-time, site-specific environmental information to users who are both remote and local to the site. A long-term goal will be to develop mobile data capture systems that can be easily deployed or activated in any environment.

Until recently, environmental data collection has been dependent on non-real-time input from installation of expensive and fragile data logging equipment and/or intermittent onsite field note collection by researchers. Now, with the aid of high-bandwidth wireless Internet connections, inexpensive sensing devices, and high-resolution tracking technologies, site-specific 
environmental data can be captured and analyzed in real-time. In addition, this data also can be made available in real-time to users while they are exploring a specific site, as well as to a wider audience of both professional and non-professional users. The WEM project is developing an integrated "environmental data gathering system" with three primary subsystems.

\subsection{Data Capture System}

A network of wireless real-time sensor stations are being installed to develop another kind of site-specific layer of information that continuously adds to the database a range of basic environmental data such as air-quality, pressure, moisture, air and soil temperature, rainfall, wind activity, fluctuation of light/dark, electromagnetic radiation, etc. In addition, sites are also being documented with audiovisual media on a regular schedule and from a grid of specific viewpoints in order to record and archive changes in the site over time.

\subsection{Database system}

This database system receives and archives the multimedia information gathered by the Data Capture System. It also contains existing imagery and data about the test site such as satellite imagery, aerial photographs, IR imagery, topographic maps, and personal photographs collected in order to compile a comprehensive, site-specific database of information.

\subsection{Visualization Authoring System}

The visualization authoring system is a $3 \mathrm{D}$ web-based interface to preview data visualizations related to a specific site, and to author placement and visualization specifications for a user browsing the site with the Wearable Environmental Media System. The system is based on an interactive $3 \mathrm{~d}$ computer generated model of the site into which users can insert spatially correspondent media objects and data visualizations. These annotation tools are designed so that a wide range of users such as scientific domain experts, artists, or even any user with a mobile phone, can "post" information to a particular site. 


\section{Future Developments and Objectives}

The intent of this research is to enable a user to easily access embedded location-specific information in any site in order to make it a more "context-rich" experience. The continuing objectives are:

- To assist, enhance, and amplify a user's interactions with the physical world though use of environmental media.

- To develop innovative interface techniques and authoring tools for the development, display, and access of location-linked virtual environments.

- To explore the concept of "context visualization" and to develop design guidelines on how to make explicit, and display for a mobile user, the layers of information and digital data that are attached to objects, people, places, as well as information about the relationships between them.

A long-term goal is to evaluate the unique advantages and opportunities that Wearable Environmental Media can provide as a learning technology in such applications areas as virtual fieldtrips, ecological literacy, environmental perception, and context visualization. Eventually, we hope this direction of research can provide the foundation for a new kind of educational infrastructure where anyone might be able to use a device like a mobile phone to ask questions about things in the environment around them wherever they are.

\section{Acknowledgements}

This research is supported in part by the Ministry of Education's Center of Excellence at Keio University and by NTT DoCoMo, Inc. Additional support for the GPS hardware has been provided by Open Network Co, Ltd., and for software development by Kawashima Lab Co., Ltd. Also thanks to Prof. Kenji Kohiyama for his support and research collaboration. 


\section{References}

[1] Milgram, P., Takemura, H., Utsumi, A., Kishino, F., "Augmented Reality: A Class of Displays on the Reality-Virtuality Continuum", SPIE Vol. 2351 Telemanipulator and Telepresence Technologies, 1994.

[2] Lippman, Andrew, "Movie-Maps: An Application of the Optical Videodisc to Computer Graphics", Computer Graphics 14(3), 1980.

[3] http://www.blackrockforest.org/

[4] http://okmesonet.ocs.ou.edu/overview/

[5] http://sensorwebs.jpl.nasa.gov/

[6] Fisher, Scott S., “ Environmental Media: Linking Virtual Environments to the Physical World”, Proceedings of the Second International Symposium on Mixed Reality, Yokohama, Japan, March, 2001

[7] Knowlton,K.C., “Computer displays optically superimposed on input devices”, Bell System Technical Journal, vol. 56,1977 , pp. $367-83$.

[8] http://www.mr-system.co.jp/project/aquagauntlet/index.html

[9] http://www.hitl.washington.edu/research/shared_space/

[10] Pulli,Petri, Pyssysalo,T., Kuutti,K., Similä,J, Metsävainio,J-P, and Komulainen,O.., “CyPhone-Future Personal Telecooperation Device", Proceedings of the XV IFIP World Computer Congress, Vienna, Austria and Budapest, Hungary, August 31 - September 4, 1998, p. 529-540.

[11] http://www.wem.sfc.keio.ac.jp/wem/applications.shtml

[12] MPEG video clip available at: http://www.wem.sfc.keio.ac.jp/wem/media.shtml 